\title{
Practice Spotlight: A Telepharmacy Model of Care for Hospitals
}

\author{
Kevin McDonald, BScPhm \\ Manager of Hospital Pharmacy \\ NorthWest Company \\ Health Products \& Services \\ Deep River, Ontario
}

\section{INTRODUCTION}

W eeneebayko General Hospital in Moose Factory, Ontario, is a 30-bed acute care hospital that serves the 9000 residents living on the James Bay coast of Ontario. Most of the population consists of Native Canadians living in 6 communities, 4 of which are accessible only by plane or by boat. Traditionally, the hospital pharmacy was to be staffed with one full-time hospital pharmacist. There are also 2 community pharmacies in the region, 1 in each of the larger communities of Moosonee and Moose Factory.

Over the years, the Weeneebayko General Hospital had trouble recruiting and retaining personnel for the hospital pharmacist position, and in 2004, the hospital again found itself with no pharmacist in this position. Although arrangements have sometimes been made for a consultant pharmacist when the pharmacist position was vacant, care was compromised during these periods of nonavailability. In 2004, in an attempt to find a long-term solution, a telepharmacy model of care was implemented. Kevin McDonald, a pharmacist who was familiar with the hospital and its staff through previous work on site, started serving the hospital from a home office in a different city, supplemented by twice-yearly on-site visits.

Telepharmacy can be defined as the use of electronic information and communications technologies to provide and support pharmaceutical care and distribution of medications when distance separates the pharmacist from the hospital. The model was designed with the intention of duplicating as many of the services that a pharmacist would provide if he or she were on site as possible.

\section{ORDER REVIEW AND ENTRY}

Under the telepharmacy model, the pharmacist reviews all medication orders for the hospital's inpatient population. After a physician writes a medication order, the order is scanned and saved in .pdf format on the hospital's secure network. The pharmacist accesses the network via the Internet, using a secure virtual private network (VPN) connection, to review the order and compare it with the computerized patient profile. The pharmacist undertakes all appropriate interventions with respect to the orders, including clarifying unclear or illegible orders, making recommendations for compliance with the hospital formulary, checking for drug interactions, confirming instructions and providing medication warnings for nurses, recommending automatic therapeutic interchanges, and ensuring that no restricted abbreviations are used. The pharmacist then enters each order into the pharmacy's computer system, and the medication labels are printed automatically in the hospital's pharmacy department. At that point, a technician-check-technician system is used to fill the order. Prescriptions are filled for individual patients, and a 7-day supply is the standard quantity.

\section{COGNITIVE PATIENT SERVICES}

The pharmacist may be asked to counsel patients on the wards before discharge. In this situation, the most reliable connection to the patient is via the telephone. The pharmacist attends the hospital's weekly multidisciplinary rounds by conference call. This allows the pharmacist to hear about the progress of individual cases and to make suggestions to team members.

\section{PHARMACY AND THERAPEUTICS INITIATIVES}

The pharmacist is active on the Pharmacy and Therapeutics Committee. Many medication safety initiatives have been implemented at the hospital since implementation of the telepharmacy program, including standardized order sets, restricted abbreviations, and "tall man" lettering.

The pharmacist works closely with the primary care team based in the hospital. One issue identified by the team has been less-than-ideal monitoring of international normalized ratios (INRs) and adjustment of warfarin dosages for the more than 70 patients in the region who are taking this drug. Another 
problem has been continuity of care, given that the physician staff is constantly changing. The telepharmacist now tracks all INR results and warfarin doses for the region in a central database, using Dawn AC software (4S Information Systems Limited, Milnthorpe, England) for anticoagulation management. As such, the pharmacist is the point of contact for all care providers and patients with respect to warfarin dosing. Since this pharmacist-monitored program was started 18 months ago, it has been recognized by physicians for improving care, and staff and patients are happy with the service.

\section{EFFECTS ON HUMAN RESOURCES}

Canada has had a long-standing shortage of pharmacists. Given the competition between community pharmacies (which often have extended hours) and hospitals for limited human resources, many pharmacist vacancies exist in hospitals across the country. More specifically, the Hospital Pharmacy in Canada 2007/2008 Report ${ }^{1}$ documented 312 hospital pharmacist vacancies (both staff and management) in Canadian hospitals and noted that a further 257 hospital pharmacists were expected to retire over the next 5 years.

That report covered hospitals with more than 50 acute care beds. However, a substantial number of smaller hospitals across the country also serve acutely ill patients but may not have the patient numbers and financial or human resources to justify employment of a full-time hospital pharmacist. Furthermore, although licensing requirements are the same for all pharmacists, regardless of whether they are working in a retail or hospital environment, today's hospitals usually benefit from employment of a pharmacist with specific hospital experience.

The main advantage of the telepharmacy model is its efficiency in utilizing limited pharmacist resources. Through telepharmacy, hospitals of any size in any geographic location can obtain the services of an experienced hospital pharmacist. Several small hospitals might even share a single telepharmacist. Such hospitals would have access to their pharmacist by phone and Internet for an 8-h workday, but would need a contract of just several hours a day to cover their needs.

\section{REGULATORY ISSUES}

The remote model of pharmaceutical care described here was initiated in Ontario for a single hospital with a distinct need. The Ontario College of Pharmacists was asked to review and to provide feedback on the model before implementation. In fact, there were no regulatory issues, since the College has no jurisdiction over hospitals in the province. Similar telepharmacy models exist elsewhere in Canada, but regulatory control over hospitals does vary by province. Some colleges may have to adjust their regulations to allow telepharmacy services in hospitals. Nonetheless, this Ontario experience has shown that a telepharmacist can effectively fulfill all of the usual responsibilities of a hospital pharmacist.

\section{EXPANSION AND FUTURE DIRECTIONS}

After its inception in 2004 in Moose Factory, the telepharmacy model of care was acquired by the NorthWest Company (Winnipeg, Manitoba) and has been extended to 10 health care institutions in Ontario and Saskatchewan, ranging in size from outpatient clinics with no beds to hospitals exceeding 400 beds (where the telepharmacist helps pharmacists working on site). Ward stock, traditional, and unit-dose systems have all been adapted to incorporate the telepharmacist's contribution. All of the hospitals being served by the NorthWest Company's Telepharmacy Solutions have been assigned experienced hospital pharmacists. At the time of writing, in late 2009, 17 pharmacists were employed to provide telepharmacy services.

Accreditation Canada now asks hospitals whether arrangements have been made for pharmacist review of all orders written while the hospital pharmacy is closed. According to the 2007/2008 report of the Hospital Pharmacy in Canada Survey, only $6 \%$ of hospitals had 24-h review of medications by a pharmacist. ${ }^{1}$ With telepharmacy, review by a pharmacist with hospital experience would be easily attainable $24 \mathrm{~h}$ a day, 7 days a week, despite limited pharmacist resources across Canada.

\section{Reference}

1. Babich M, Bussières JF, Hall KW, Harding J, Johnson N, Lefebvre P, et al., editors. Hospital pharmacy in Canada 2007/2008 report. Eli Lilly; 2008. Available from: http://www.lillyhospitalsurvey.ca/hpc2/content/rep_2008_ toc.asp
The Practice Spotlight series highlights the accomplishments of Canadian pharmacists with unique practices in hospitals and related health care settings. If you have a unique or innovative practice, or you know someone else who should be profiled, please submit your contact information to Mary Ensom, Editor of CJHP (cjhpedit@cshp.ca), and one of our Associate Editors will be in touch with you. 